\title{
miR-101, downregulated in retinoblastoma, functions as a tumor suppressor in human retinoblastoma cells by targeting EZH2
}

\author{
QIONG LEI $^{1}$, FENGMEI SHEN $^{1}$, JIE WU $^{1}$, WEISHAN ZHANG ${ }^{2}$, \\ JIAHUI WANG $^{2}$ and LIN ZHANG ${ }^{1}$ \\ ${ }^{1}$ Department of Ophthalmology, First Affiliated Hospital, Xi'an Jiaotong University; \\ ${ }^{2}$ Department of Ophthalmology, No.1 Hospital of Xi'an, Xi'an, Shaanxi 710062, P.R. China
}

Received January 26, 2014; Accepted March 29, 2014

DOI: $10.3892 / o r .2014 .3167$

\begin{abstract}
Accumulating evidence indicates that microRNAs are involved in multiple processes in cancer development and progression, and several miRNAs have emerged as candidate components of oncogene or tumor-suppressor networks in retinoblastoma. miR-101 has been identified as a tumor suppressor in several types of human cancer. However, the specific function of miR-101 in retinoblastoma remains unclear. In the present study, we found that the expression of miR-101 in retinoblastoma tissues was much lower than that in the normal controls. In addition, downregulation of miR-101 more frequently occurred in retinoblastoma specimens with adverse clinicopathological and histopathological features. In addition, miR-101 inhibited cell viability and progression in retinoblastoma cells by promoting cell apoptosis and arresting the cell cycle. Finally, we found that miR-101 directly inhibited EZH2 expression by targeting its 3'-UTR, and EZH2 was upregulated and inversely correlated with miR-101 expression in the retinoblastoma tissues. Thus, for the first time, we provide convincing evidence that downregulation of miR-101 is associated with tumor aggressiveness in retinoblastoma and inhibits cell growth and proliferation of retinoblastoma cells by targeting EZH2. In conclusion, all the evidence supports the tumor-suppressor role of miR-101 in human retinoblastoma.
\end{abstract}

Correspondence to: Professor Lin Zhang, Department of Ophthalmology, First Affiliated Hospital, Xi'an Jiaotong University, 227 YanTa West Road, Xi'an, Shaanxi 710062, P.R. China

E-mail: zhanglinxjtu@163.com

Abbreviations: RB, retinoblastoma; miR-101, microRNA-101; $\mathrm{EZH} 2$, enhancer of zeste homolog 2; PRC2, polycomb repressive complex 2; WT, wild-type; MT, mutant-type; 3'-UTR, 3'-untranslated region; qRT-PCR, quantitative real-time polymerase chain reaction; $\mathrm{PD}$, poor differentiation; WD, well differentiation

Key words: miR-101, retinoblastoma, proliferation, cell cycle, cell apoptosis, EZH2

\section{Introduction}

Retinoblastoma (RB) is the most common primary intraocular malignancy in children worldwide, with a prevalence ranging from $1: 15,000$ to $1: 20,000$ in children under the age of 5 years and with an estimated 2,800 new cases in the US $(1,2)$. Both forms of this malignancy, hereditary and sporadic, are associated with alteration or loss of the tumor-suppressor gene RB1. The RB1 protein (pRB) functions as a tumor suppressor by controlling the cell cycle and differentiation via complex interaction with multiple kinases and their inhibitors $(3,4)$. Evidence from many studies suggests that several genes and pathways are dysregulated and misexpressed in RB, and the expression of RB1 can be modulated (5). Thus, understanding the molecular mechanisms of RB progression more thoroughly will undoubtedly benefit the development of new therapeutic strategies.

MicroRNAs are small noncoding RNAs, 18-25 nucleotides in length, transcribed from non-protein-coding genes or introns. They regulate gene expression by repressing translation and cleaving their target mRNAs by binding to complementary sites in their 3'-untranslated region (UTR). It has been demonstrated that due to aberrant expression, miRNAs may function as tumor suppressors or oncogenes according to the roles of their target gene $(6,7)$. Particularly, miRNAs can regulate various cellular processes of tumor cells that involve the cell cycle, differentiation, progression, apoptosis, proliferation, migration and invasion (8-10). A number of miRNAs have been proven to induce proliferation by targeting members of the pRB family (11). Moreover, several miRNAs have emerged as candidate components of oncogene or tumor suppressor networks in RB, such as carcinogenic miR-17 92, and tumor suppressor miR-365-2p, miR-34a and let-7 (12-15).

miR-101 as a tumor suppressor has recently attracted much attention. It has been reported that the expression of miR-101 was downregulated during prostate cancer progression, and the expression of EZH2 was upregulated concomitantly during this process (16). Enhancer of zeste homolog 2 (EZH2), a histone methyltransferase, is a catalytic subunit of a Polycomb repressive complex 2 (PRC2) which methylates histone $\mathrm{H} 3$ on lysine 27 (17). Mounting evidence proves that EZH2 has properties consistent with those of an oncogene, since overexpression of EZH2 promotes cell proliferation, colony formation 
and enhanced cell migration and invasion $(18,19)$. The involvement of miR-101 in carcinogenesis through downregulation of EZH2 has been subsequently confirmed in bladder cancer, lung cancer, gastric cancer, hepatocellular cancer and glioblastoma (20-26). However, the function of miR-101 and EZH2 in $\mathrm{RB}$ has not yet been thoroughly investigated.

In the present study, we investigated the expression of miR-101 and EZH2 in RB patients by quantitative real-time polymerase chain reaction (qRT-PCR). We reported that miR-101 was significantly downregulated in RB patient tissues compared to normal control retina, which was accompanied by the upregulation of EZH2 in RB tumor tissues. Furthermore, we evaluated the association between the expression of miR-101 and EZH2 with histopathological and clinicopathological features. Finally, miR-101 was identified to inhibit the expression of EZH2 directly and function as a tumor suppressor by inhibiting cell growth and inducing cell cycle arrest and cell apoptosis. To the best of our knowledge, this is the first comprehensive and thorough study to exam the expression and function of miR-101 and its target EZH2 in RB.

\section{Materials and methods}

Patients and tissue samples. This study was approved by the Research Ethics Committee of Xi'an Jiaotong University. Written informed consent was obtained from all of the patients. All specimens were handled and made anonymous according to the ethical and legal standards. A total of $87 \mathrm{RB}$ tissues and 44 normal retinas were provided by the Department of Ophthalmology, the First Affiliated Hospital, Xi'an Jiaotong University. The ages of the subjects ranged from 1 month to 14 years (median, 28 months). Among the patients, 52 (59.8\%) were affected unilaterally and $35(40.2 \%)$ bilaterally. One tumor from each patient with bilateral tumors was used in this study. Histopathological analysis revealed that $66(75.9 \%)$ of the tumors were poorly differentiated (PD) and 21 (24.1\%) were well differentiated (WD). Invasion of the choroid, optic nerve or orbit was detected in $52(59.8 \%)$ tumors, while $35(40.2 \%)$ tumors were localized without invasion. Among the 52 invasive tumors, 39 exhibited choroidal invasion, 37 exhibited optic nerve invasion and 32 exhibited orbital invasion.

Quantitative reverse transcriptase PCR ( $q R T-P C R)$ assay. The expression levels of miR-101 in RB and normal retinal tissues were detected by qRT-PCR assay. Briefly, total RNA was extracted from tissues using TRIzol reagent (Invitrogen, Carlsbad, CA, USA) according to the manufacturer's protocol. miRNA expression levels were then quantitated using TaqMan miRNA real-time RT-PCR kit (Applied Biosystems) according to the manufacturer's protocol. Data were analyzed using 7500 software v.2.0.1 (Applied Biosystems), with the automatic Ct setting for adapting baseline and threshold for $\mathrm{Ct}$ determination. The universal small nuclear RNA U6 (RNU6B) was used as an endogenous control for miRNAs. Each sample was examined in triplicate, and the amounts of PCR products produced were nonneoplasticized to RNU6B.

Cell culture. Human RB cell lines Y79 and WERI-RB1 were obtained from the Cell Bank of the Chinese Academy of
Sciences (Shanghai, China), where they were characterized by mycoplasma detection, DNA-fingerprinting, isozyme detection and cell vitality detection. They were cultured in DMEM (Invitrogen, Carlsbad, CA, USA) supplemented with 10\% fetal bovine serum (FBS)(HyClone, Logan, UT, USA) and cultured in a humidified incubator at $37^{\circ} \mathrm{C}$ in $5 \% \mathrm{CO}_{2}$.

Oligonucleotide transfection. miR-101 mimics and inhibitors were chemically synthesized by Shanghai GenePharma (Shanghai, China). Once the cells were $80 \%$ confluent, miR-101 mimics or the inhibitor was transfected into the RB cells with Lipofectamine 2000 (Invitrogen) according to the manufacturer's instructions. Cells were also transfected with a scramble oligonucleotide as negative control (NC). The expression level of miR-101 in the transfected RB cells was determined by qRT-PCR.

Luciferase reporter assay. RB cells were seeded in a 96-well plate at $60 \%$ confluency. After $24 \mathrm{~h}$, cells were transfected with $120 \mathrm{ng}$ of miR-101 expression vector or negative control. Cells were transfected with $30 \mathrm{ng}$ of WT or MT 3'-UTR of EZH2 mRNA. Cells were collected $48 \mathrm{~h}$ after transfection, and luciferase activity was measured using a dual luciferase reporter assay system according to the manufacturer's protocol (Promega).

Cell viability assay. Cells were plated in 96-well plates $\left(0.5 \times 10^{4}\right.$ cells/well) and transfected with NC, miR-101 mimics and inhibitors. Forty-eight hours later, $10 \mu \mathrm{l}$ of MTT reagent $(5 \mathrm{mg} / \mathrm{ml})$ was added to each well, and cells were incubated at $37^{\circ} \mathrm{C}$ for another $4 \mathrm{~h}$. The medium was removed, the cells were solubilized in $150 \mu \mathrm{l}$ of dimethylsulfoxide, and colorimetric analysis was performed (wavelength, $490 \mathrm{~nm}$ ). One plate was analyzed immediately after the cells adhered $(\sim 4 \mathrm{~h}$ after plating), and the remaining plates were assayed every day for the next 4 consecutive days.

Soft agar colony formation assay. Cells seeded on a 6-well plate were covered with a layer of $0.6 \%$ agar in DMEM supplemented with $10 \%$ FBS. After transfection for $48 \mathrm{~h}$, cells were trypsinized, gently mixed with $0.3 \%$ agar medium mixture containing selective antibiotics and reseeded in triplicate onto a 6-well plate. After 4 weeks, the resistant colonies were stained with $0.2 \%$ crystal violet and counted under a microscope.

Flow cytometric analysis of cell cycle distribution and cell apoptosis. The RB cells were transfected with NC, miR-101 mimics or miR-101 inhibitors. Forty eight hours post-transfection, cells were trypsinized and analyzed for cell cycle distribution and cell apoptosis. For cell cycle distribution, half of the cells of each group was stained with propidium iodide (PI) and analyzed by flow cytometry using FACSCalibur (BD Biosciences, San Diego, CA, USA). For each group, 10,000 events were acquired. The percentages of cells in the G1, S and $\mathrm{G} 2$ phases of the cell cycle were calculated. The other half of cells of each group was used to detect cell apoptosis using Annexin V-FITC and PI (BD Bioscience, USA) following the manufacturer's instructions. The apoptosis index was calculated by adding the cells in the first and the cells in the second group. 
A
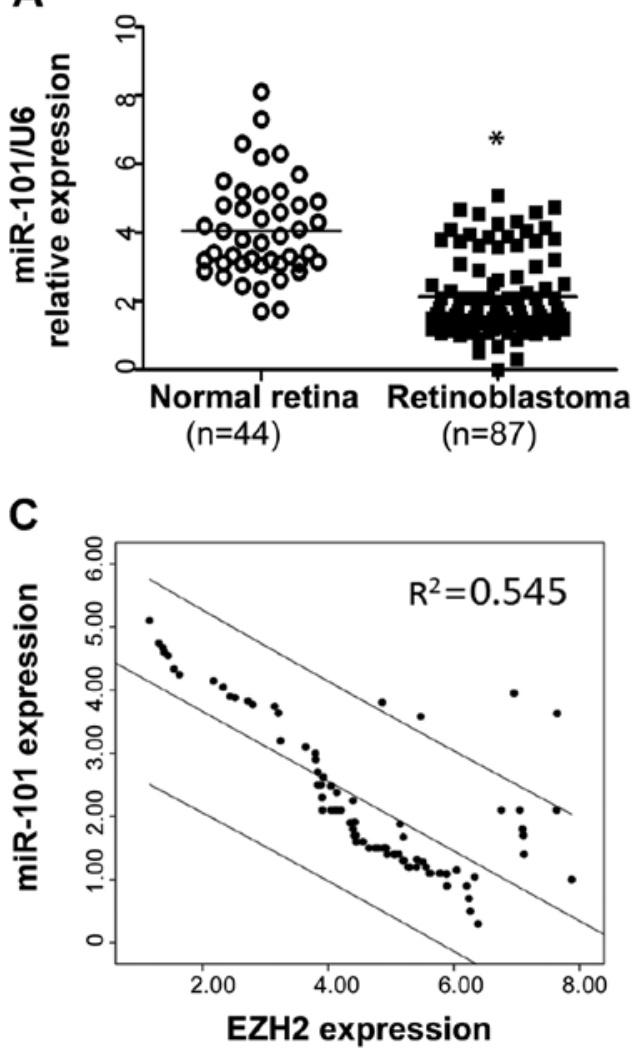

B

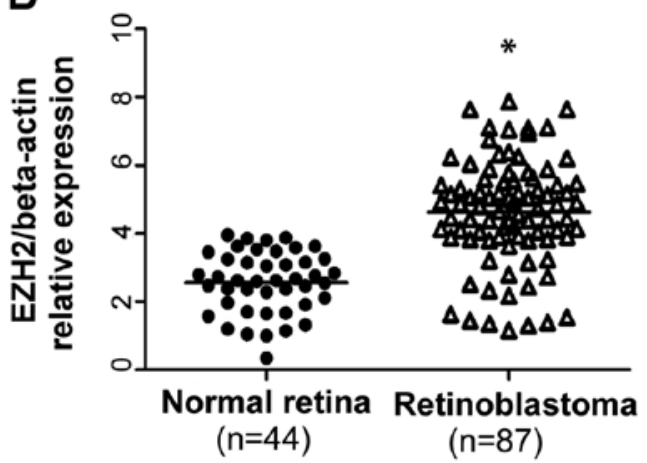

D

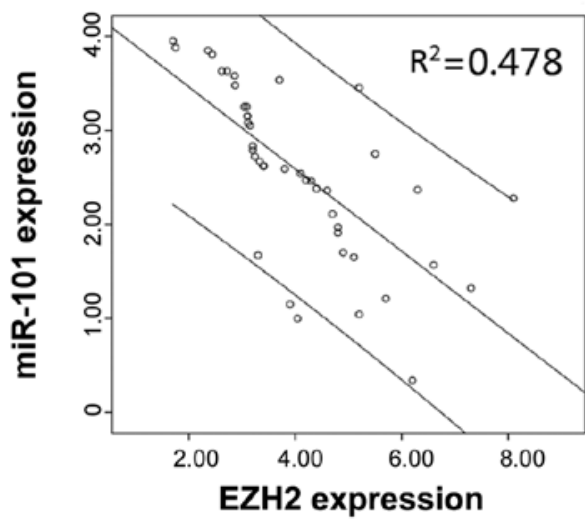

Figure 1. Expression levels of miR-101 and EZH2 in retinoblastoma and normal retina. (A) The relative expression of miR-101 in 87 human retinoblastoma and 44 normal retina samples. (B) The relative expression of EZH2 in 87 human retinoblastoma and 44 normal retina samples. (C) The expression of miR-101 was negatively correlated with the expression of EZH2 in the retinoblastoma tissues. (D) The expression of miR-101 was negatively correlated with the expression of EZH2 in the normal retina samples. Expression levels of miR-101 and EZH2 were determined by qRT-PCR and normalized against an endogenous control U6 RNA and $\beta$-actin, respectively. ${ }^{*} \mathrm{P}<0.05$.

Statistical analysis. Statistical analysis was performed using IBM SPSS statistical software (version 20.0). The differences in characteristics between the 2 groups were examined by the $\chi^{2}$ test or Fisher's exact test. All P-values were determined from 2-sided tests, and statistical significance was based on a P-value of 0.05 .

\section{Results}

Expression of miR-101 and EZH2 in nontumor retina samples and retinoblastomas. Expression levels of miR-101 and EZH2 were analyzed in 44 normal retina samples and 87 tumor samples by quantitative real-time polymerase chain reaction (qRT-PCR) and normalized against endogenous controls (U6 RNA or $\beta$-actin, respectively). As shown in Fig. 1A, the expression level of miR-101 in RB tissues was found to be distinctly downregulated compared to the nontumor retina tissues. In contrast, weak or absent EZH2 expression was detected in the nontumor retina tissues, but abundant expression of EZH 2 was detected in the RB tissues (Fig. 1B). Furthermore, we detected an association among miR-101 expression and EZH2 in these nontumor retina and RB cases. As shown in Fig. $1 \mathrm{C}$ and $\mathrm{D}$, statistically significant inverse correlations were revealed by Spearman's correlation analysis between mRNA levels of miR-101 and EZH2 in RB ( $\mathrm{r}=-0.738$; $\mathrm{P}<0.001)$ and nontumor retina tissues $(r=-0.691 ; \mathrm{P}<0.001)$. Taken together, our result suggests that miR-101 plays a tumor-suppressor role and EZH2 plays an oncogenic role in RB. More importantly, miR-101 was negatively correlated with the expression of EZH2 in both the normal retina and $\mathrm{RB}$.

Relationship between miR-101/EZH2 and clinicopathological features of the retinoblastoma cases. To determine the clinical significance of miR-101 and its potential target EZH2 in RB, we analyzed the association of miR-101 and EZH2 with various clinicopathological and histopathological parameters of the RB cases. The median miR-101 and EZH2 expression levels in the 87 patients of RB were 2.74 and 4.72, respectively. The patients were divided into two groups according to their expression levels of miR-101 and EZH2, using its median as a cut off: high miR-101 expression group $(n=34)$ and low miR-101 expression group ( $n=53)$. Accordingly, a high EZH2 expression group $(n=46)$ and a low EZH2 expression group $(n=41)$ were established. As shown in Table IA, a significant association was found between low miR-101 expression and the degree of invasion, subretinal and vitreous seeding $(\mathrm{P}=0.045$, $\mathrm{P}=0.001$ and $\mathrm{P}=0.007$, respectively). Whereas, $\mathrm{EZH} 2$ was significantly upregulated in $\mathrm{RB}$ patients with invasion, subretinal and vitreous seeding $(\mathrm{P}=0.018, \mathrm{P}=0.004$ and $\mathrm{P}=0.029$, respectively). No significant differences were observed in the miR-101 and EZH2 expression levels in regards to patient age, gender and laterality. 
Table I. Association of miR-101 and EZH2 expression with clinicopathological and histopathological features of the retinoblastoma cases.

\section{A, Clinicopathological features}

\begin{tabular}{|c|c|c|c|c|c|c|c|}
\hline & \multirow[b]{2}{*}{$\begin{array}{c}\text { No. } \\
\text { of cases }\end{array}$} & \multicolumn{2}{|c|}{ miR-101 expression } & \multirow[b]{2}{*}{ P-value } & \multicolumn{2}{|c|}{ EZH2 expression } & \multirow[b]{2}{*}{ P-value } \\
\hline & & $\begin{array}{c}\text { High } \\
\text { n }\end{array}$ & $\begin{array}{c}\text { Low } \\
n\end{array}$ & & $\begin{array}{c}\text { High } \\
\mathrm{n}\end{array}$ & $\begin{array}{c}\text { Low } \\
\mathrm{n}\end{array}$ & \\
\hline Age (years) & & & & 0.826 & & & 0.287 \\
\hline$<2$ & 48 & 18 & 30 & & 28 & 20 & \\
\hline$\geq 2$ & 39 & 16 & 23 & & 18 & 21 & \\
\hline Gender & & & & 0.829 & & & 0.394 \\
\hline Male & 45 & 17 & 28 & & 26 & 19 & \\
\hline Female & 42 & 17 & 25 & & 20 & 22 & \\
\hline Laterality & & & & 0.655 & & & 0.284 \\
\hline Unilateral & 52 & 19 & 33 & & 30 & 22 & \\
\hline Bilateral & 35 & 15 & 20 & & 16 & 19 & \\
\hline Invasion & & & & $0.045^{\mathrm{a}}$ & & & $0.018^{\mathrm{a}}$ \\
\hline Noninvasive & 35 & 9 & 26 & & 13 & 22 & \\
\hline Invasive & 52 & 25 & 27 & & 33 & 19 & \\
\hline Subretinal seeding & & & & $0.001^{\mathrm{a}}$ & & & $0.004^{\mathrm{a}}$ \\
\hline Yes & 31 & 5 & 26 & & 23 & 8 & \\
\hline No & 56 & 29 & 27 & & 23 & 33 & \\
\hline Vitreous seeding & & & & $0.007^{\mathrm{a}}$ & & & $0.029^{\mathrm{a}}$ \\
\hline Yes & 37 & 8 & 29 & & 25 & 12 & \\
\hline No & 50 & 26 & 24 & & 21 & 29 & \\
\hline
\end{tabular}

B, Histopathological features

\begin{tabular}{|c|c|c|c|c|c|c|c|}
\hline Differentiation & & & & $0.004^{\mathrm{a}}$ & & & $0.013^{\mathrm{a}}$ \\
\hline WD & 21 & 14 & 7 & & 6 & 15 & \\
\hline $\mathrm{PD}$ & 66 & 20 & 46 & & 40 & 26 & \\
\hline Necrosis & & & & 0.579 & & & 0.300 \\
\hline None & 38 & 13 & 25 & & 23 & 15 & \\
\hline Mild & 14 & 5 & 9 & & 8 & 6 & \\
\hline Extensive & 35 & 16 & 19 & & 15 & 20 & \\
\hline Optic nerve invasion & & & & $0.026^{\mathrm{a}}$ & & & 0.082 \\
\hline Yes & 37 & 9 & 28 & & 24 & 13 & \\
\hline No & 50 & 25 & 25 & & 22 & 28 & \\
\hline Choroidal invasion & & & & $0.008^{\mathrm{a}}$ & & & $0.009^{\mathrm{a}}$ \\
\hline Yes & 39 & 9 & 30 & & 27 & 12 & \\
\hline No & 48 & 25 & 23 & & 19 & 29 & \\
\hline Orbital invasion & & & & $0.044^{\mathrm{a}}$ & & & $0.028^{\mathrm{a}}$ \\
\hline Yes & 32 & 8 & 24 & & 22 & 10 & \\
\hline No & 55 & 26 & 29 & & 24 & 31 & \\
\hline
\end{tabular}

${ }^{\mathrm{a}} \mathrm{P} \leq 0.05$. PD, poorly differentiated; WD, well differentiated.

Relationship between miR-101/EZH2 and histopathological features of retinoblastoma. The possible association between miR-101/EZH2 and histopathologic features was then analyzed. As shown in Table IB, miR-101 was significantly downregulated in $\mathrm{RB}$ cases with poor differentiation (PD) compared to cases with well differentiation (WD) $(\mathrm{P}=0.004)$, 
A

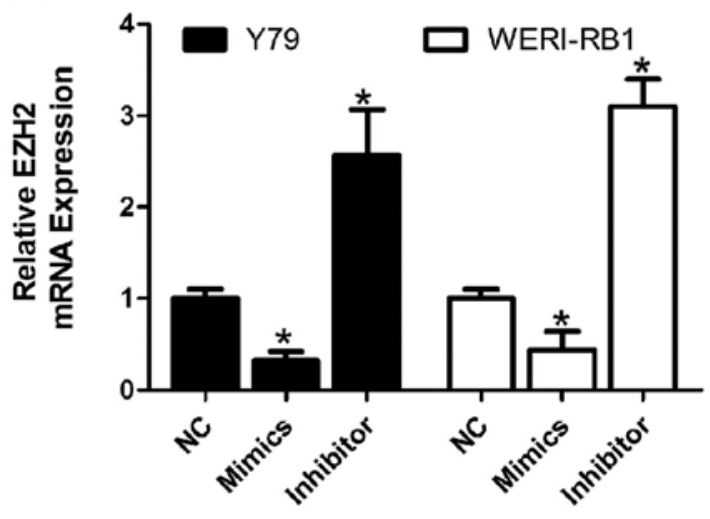

C

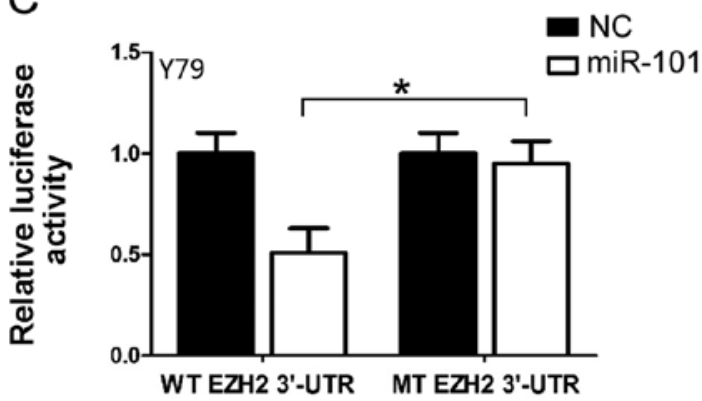

B
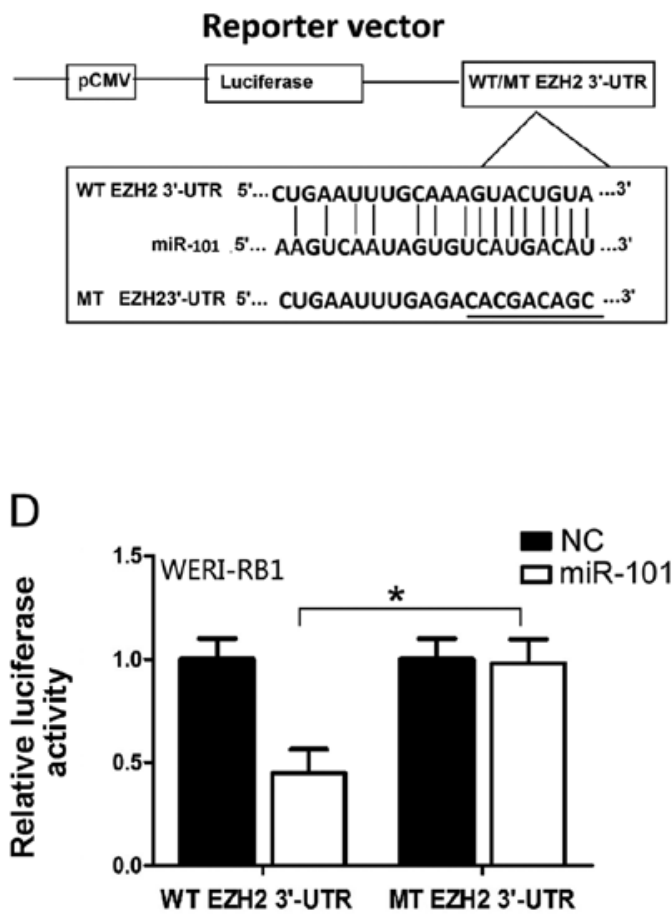

Figure 2. EZH2 3'-UTR is the direct target of miR-2101. (A) Transfection of miR-101 mimics or inhibitors in Y79 and WERI-RB1 cells increased or decreased, respectively, the expression level of EZH2 in retinoblastoma. (B) miR-101 and its putative binding sequence in the 3'-UTR of EZH2, and the diagram of the luciferase reporter plasmids with WT and MT EZH2 3'-UTR. The relative luciferase activity in (C) Y79 and (D) WERI-RB1 cells after the plasmid with WT or MT EZH2 3'-UTR was co-transfected with miR-101 mimics. Three independent experiments were performed in duplicate. Data are expressed as means \pm SD. Two-tailed Student's t-test was used to analyze the significant differences. * $\mathrm{P}<0.05$. NC, normal control; 3'-UTR, 3'-untranslated region; EZH2, enhancer of zeste homolog 2; WT, wild-type; MT, mutant-type.

whereas EZH2 was significantly upregulated in RB cases with $\mathrm{PD}$ compared to WD $(\mathrm{P}=0.013)$. Moreover, the expression of miR-101 was also significantly associated with choroidal invasion $(\mathrm{P}=0.008)$, invasion of the optic nerve $(\mathrm{P}=0.026)$ and orbital invasion $(\mathrm{P}=0.044)$. EZH2 expression was significantly associated with orbital invasion $(\mathrm{P}=0.028)$ and choroidal invasion $(\mathrm{P}=0.009)$. No significant differences were observed between the other histopathological features and the expression of miR-101 and EZH2.

EZH2 is a direct target of miR-101 in retinoblastoma cells. It has been proven that EZH2 is the direct target of miR-101 in other cancer cells $(26,27)$. Considering the tissue-specific and developmental stage-specific manner of microRNAs, we investigated the relationship of EZH2 and miR-101 in RB cell lines. In order to confirm whether EZH2 was a target gene for miR-101 in RB cells, qRT-PCR was used to detect the expression of EZH2 which was regulated by miR-101 in the RB cell lines Y79 and WERI-RB1. The expression of EZH2 was significantly downregulated after overexpression of miR-101 at the mRNA level in RB cells (Fig. 2A). Furthermore, we assessed the significance of miR-101 and EZH2 correlation in the RB tissues. Previously, we determined the EZH2 mRNA and miR-101 expression in the same RB specimens by qRT-PCR. As shown in Fig. 1C, a statistically significant inverse correlation was revealed by Spearman's correlation analysis between the mRNA levels of miR-101 and EZH2 in $\mathrm{RB}(\mathrm{r}=-0.738 ; \mathrm{P}<0.001)$. Taken together, our results suggest that miR-101 negatively regulates the expression of its potential target gene EZH2 in RB.

We further performed a luciferase reporter assay to verify whether miR-101 directly targets the 3'-UTR of EZH2 in RB cells. The target sequence of EZH2 3'-UTR (WT 3'-UTR) or the mutant sequence (MT 3'-UTR) was cloned into a luciferase reporter vector (Fig. 2B). Y79 and WERI-RB1 cells were then transfected with the WT or MT 3'-UTR vector and miR-101 mimic. As shown in Fig. 2C, a significant decrease in luciferase activity was demonstrated between the EZH2 WT 3'-UTR group and the negative control group in the Y79 cells $(\mathrm{P}<0.05)$. The suppressive effect was abrogated by a point mutation in the core binding sites of the EZH2 3'-UTR. A similar trend was also found in the WERI-RB1 cells (Fig. 2D). All these results indicate that miR-101 exerts inhibitory effects on EZH2 expression via interaction with 3'-UTR of EZH2 in $\mathrm{RB}$ cells.

Effect of miR-101 on cell viability and proliferation of human retinoblastoma cell lines. It has been reported that EZH2 promotes cell proliferation, colony formation and increased cell apoptosis in many different cancer cells $(16,19,28,29)$. As the patients with adverse clinicopathological and histopathological features were significantly associated with low expression of miR-101 and EZH2 was found to be the direct target of miR-101, we investigated whether miR-101 affects cell viability, cell cycle distribution and cell apoptosis in $\mathrm{RB}$ cells. To confirm this possibility, we increased the miR-101 
A
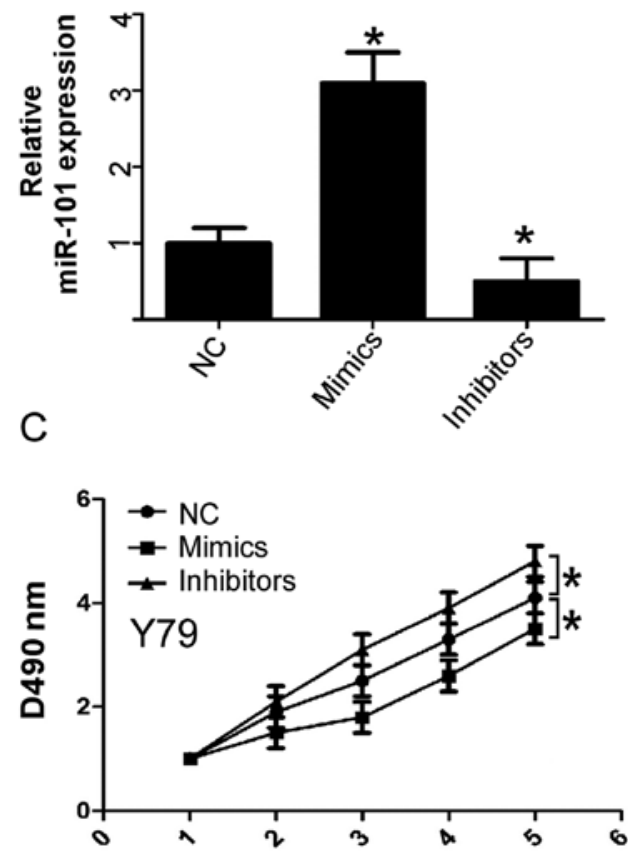

E

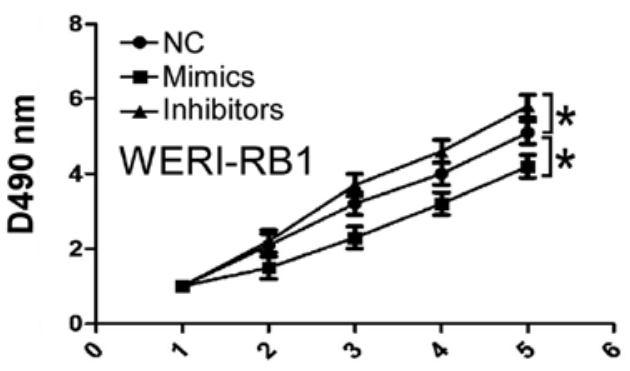

G

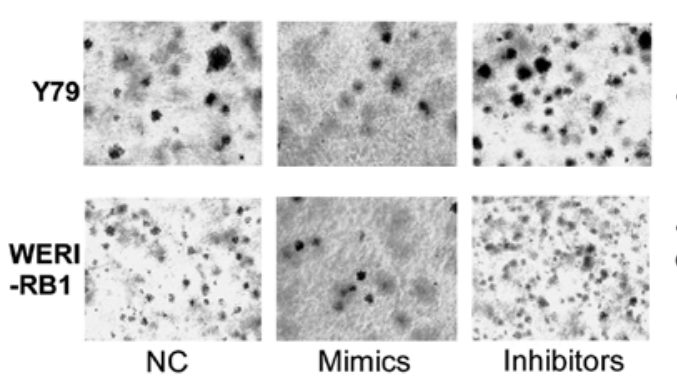

B
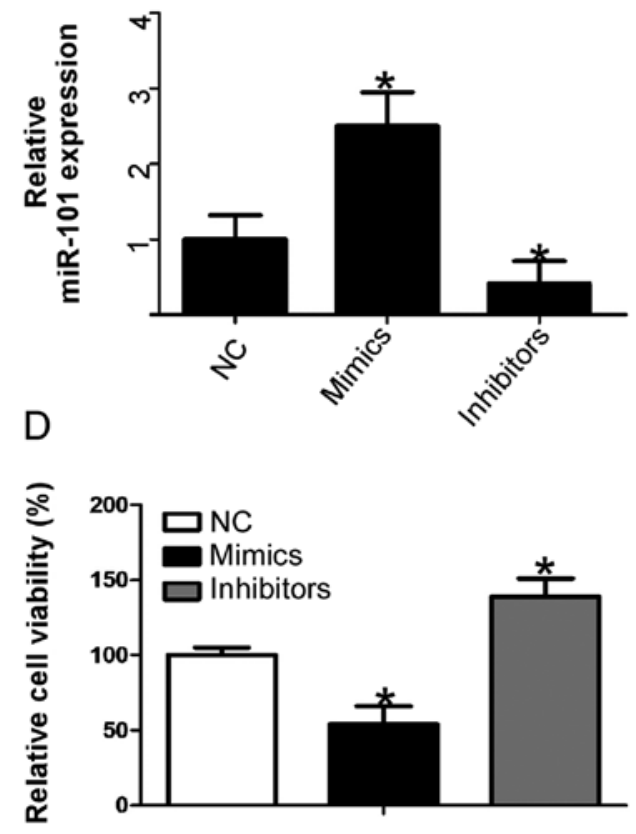

$\mathrm{F}$

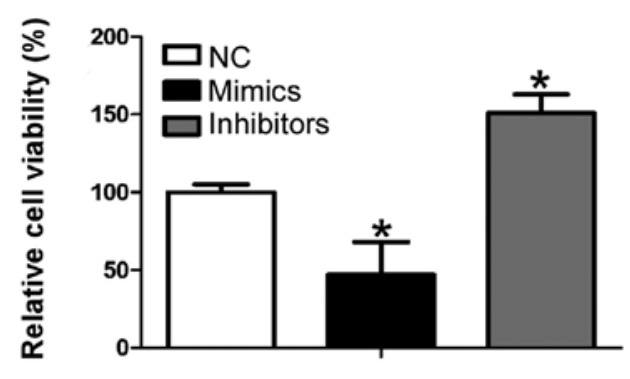

$\mathrm{H}$

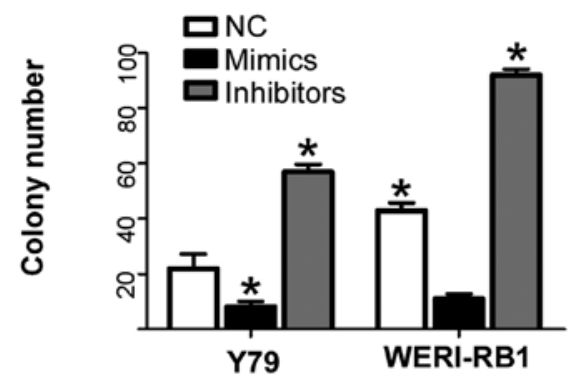

Figure 3. Associations between miR-101 expression and cell viability and cell proliferation of retinoblastoma cell lines. (A and B) Transfection of Y79 and WERI-RB1 cells with miR-101 mimics or inhibitors increased and decreased the expression of miR-101, respectively, as detected by qRT-PCR. (C-F) Effects of miR-101 on the cell viability of Y79 and WERI-RB1 cells. (G and H) Effects of miR-101 on cell proliferation of Y79 and WERI-RB1 cells. Three independent experiments were performed in duplicate. Data are present as means \pm SD. Two-tailed Student's t-test was used to analyze significant differences. ${ }^{*} \mathrm{P}<0.05$.

level in human RB cells with miR-101 mimics or decreased the miR-101 level in human RB cells with miR-101 inhibitors. Then human RB cell lines Y79 and WERI-RB-1 were transiently transfected with miR-101 mimics or inhibitors, respectively. As expected, transfection of miR-101 mimics or miR-101 inhibitors caused an increase or a decrease in miR-101 expression, respectively, compared with the negative control (Fig. 3A and B). After confirming the efficiency of the miR-101 mimics and inhibitors, we determined the effect of increased and decreased expression of miR-101 on cell viability using an MTT assay. RB cells that were transfected with miR-101 mimics showed a significant decrease in cell viability when compared to the normal control. Whereas, RB cells transfected with the miR-101 inhibitor showed a significant increase in cell viability when compared to the normal control (Fig. 3C-F). We then determined the effect of increased and decreased expression of miR-101 on cell proliferation using soft agar colony formation assays. RB cell lines transfected 
A
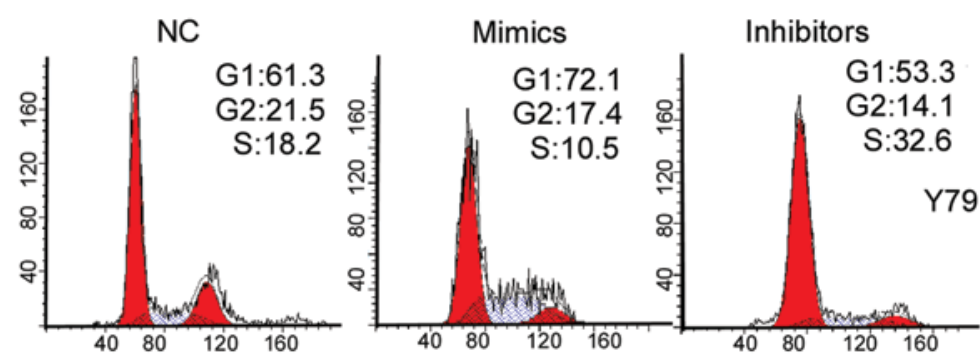

C
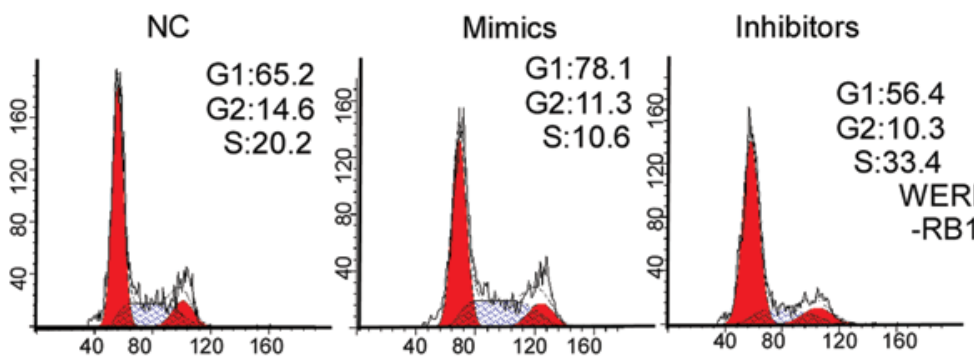

E

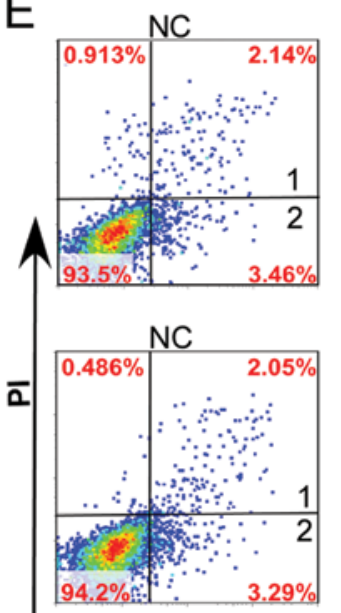

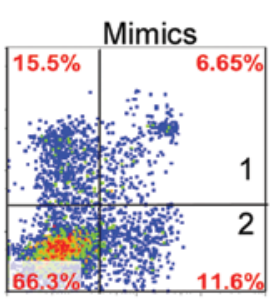

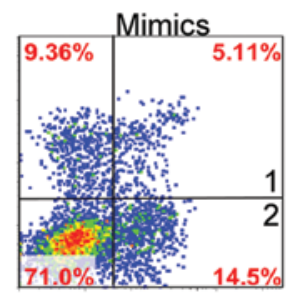

Annexin-V

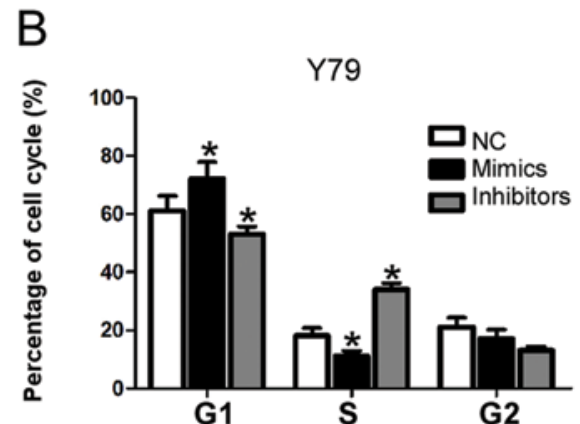

D

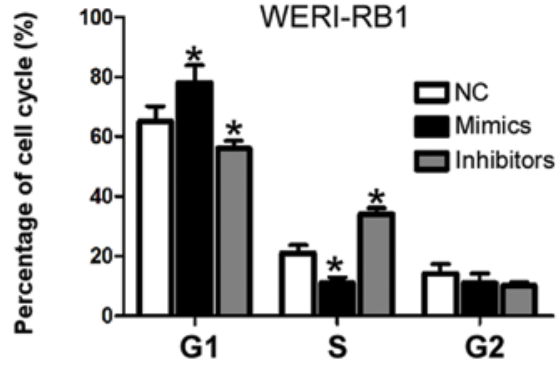

F

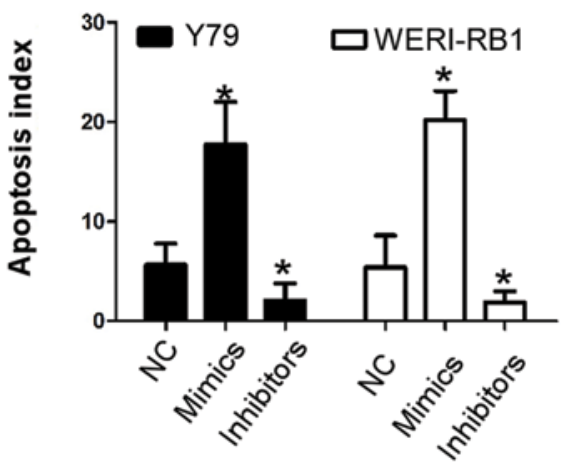

Figure 4. Association between miR-101 expression and cell cycle distribution and cell apoptosis in retinoblastoma cell lines. (A-D) The effects of miR-101 on the cell cycle of Y79 and WERI-RB1 cells. (E and F) The effects of miR-101 on cell apoptosis of Y79 and WERI-RB1 cells. Three independent experiments were performed in duplicate. Data are presented as means \pm SD. Two-tailed Student's t-test was used to analyze significant differences. $P<0.05$.

with miR-101 mimics showed a significant decrease in cell proliferation compared to the normal control, whereas, RB cells transfected with miR-101 inhibitors showed a significant increase in cell proliferation compared to the normal control (Fig. 3G and H). These data suggest that enforced expression of miR-101 can significantly suppress human RB cell growth, and decreased expression of miR-101 significantly promotes human RB cell growth, which indicates a tumor-suppressor role for miR-101 in human RB cells.

Effect of miR-101 on cell cycle and apoptosis of human retinoblastoma cell lines. To further investigate the mechanism of miR-101-induced cell growth inhibition, we assessed the change in cell cycle distribution by flow cytometry. In RB cell lines, Y79 and WERI-RB1, miR-101 mimics significantly decreased the percentages of cells in the S-phase, whereas the percentages of cells were increased in the G1-phase compared to the normal control transfected cells. whereas, miR-101 inhibitors significantly increased the percentages of cells in the S-phase and decreased the percentages of cells in the G1-phase compared to the normal control transfected cells (Fig. 4A-D). Previous studies confirmed that miR-101 is a p53-regulated microRNA, and the activation of the p53 pathway results in apoptosis in various cancer cells $(30,31)$. We hypothesized that the exogenous miR-101 administration may result in activation of an apoptotic program in RB cells. Thus, we evaluated the effect of 
miR-101 on cell apoptosis of RB cell lines. As shown in Fig 4E and $\mathrm{F}$, enhanced expression of miR-101 significantly increased the percentage of apoptotic cells, while decreased expression of miR-101 significantly decreased the apoptosis index of RB cells. Taken together, these results indicate that miR-101 induces G1 arrest and promotes cell apoptosis in RB cells.

\section{Discussion}

Several microRNAs have been identified as candidate components of oncogene and tumor-suppressor networks in RB (5), and the microRNA miR-101 has been found to be a tumor suppressor and is a prognostic marker in several types of cancer by targeting EZH2, MIF or SOX9 $(20,21,32,33)$. However, the function and clinical relevance of miR-101 in RB have not yet been studied. In the present study, we found that miR-101 was weakly expressed in RB tissues compared to normal retina tissues. At the same time, its potential target EZH2 had a higher expression level in RB tissues than in normal retina. We also found that miR-101 was inversely associated with the expression of EZH2 in normal retina and RB. Moreover, we demonstrated that decreased expression of miR-101 and increased expression of EZH2 were significantly associated with adverse clinicopathological and histopathological features. Our results showed that miR-101 was significantly associated with invasion, subretinal seeding, vitreous seeding, choroidal invasion, orbital, optic nerve invasion in RB patients. EZH2 was significantly associated with invasion, subretinal seeding, vitreous seeding, orbital invasion and choroidal invasion in the RB patients. These results provide initial evidence to support that miR-101 functions as a tumor suppressor in RB and that EZH2 is its potential target and has an oncogenic role in RB.

To reveal the role of miR-101 in RB cells, we tested the effect of miR-101 on cell growth and cell proliferation. Our results showed that ectopic expression of miR-101 significantly reduced cell growth and proliferation in RB cell lines Y79 and WERI-RB1. These results indicate that miR-101 is a novel tumor suppressor and plays important roles in the regulation of tumor proliferation of RB. Next, we investigated the potential mechanism of miR-101 in cell growth and proliferation and found that high levels of miR-101 led to cell cycle arrest and increased cell apoptosis. It has also been reported that miR-101 is downregulated in non-small cell lung cancer and hepatocellular carcinoma cell lines, resulting in the promotion of apoptosis and inhibition of cell cycle progression $(26,34,35)$. Combining these studies and our results, we confirm that miR-101 functions as a tumor-suppressor gene by affecting the cell cycle and cell apoptosis in these types of cancer.

The expression of miR-101 in cancers is still controversial. Most studies indicate that miR-101 is significantly downregulated in different types of cancers. However, one study found that miR-101 was upregulated in prostate cancer tissues (36). Our results make this controversy even more complicated. Conkrite et al (37) found that miR-101 was upregulated in human retinoblastoma compared to normal retina using microarray and deep-sequencing analysis, which was opposite to our results. The discrepancies between our study and their study concerning miR-101 expression in RB may be due to the differences in sample origin, different analytical approaches or different technical platforms of studies. In our study, we applied qRT-PCR as the main strategy to detect the expression of miR-101. Consistent with most previous studies, the expression of miR-101 was downregulated in RB compared to normal retina tissues. In their study, they examined global expression of microRNAs in human retinoblastoma using microarrays. They found that miR-101 was upregulated in RB compared to normal retina tissues, but they did not further identify their results using qRT-PCR in a larger RB sample. However, it is also possible that miR-101 has a dual role in the tumorigenicity of RB. To date, several miRNAs with a dual role in cancer have been reported. For example, miR-155 is upregulated in breast cancer, while the expression of miR-155 is significantly decreased in pancreatic tumors $(38,39)$, which indicates the dual role of miR-155 in cancer and the tissue-specific nature of microRNAs. Thus, in order to further identify the tumorsuppressor role of miR-101 in RB, we further investigated the effect of miR-101 on RB cells.

As a tumor suppressive miRNA, miR-101 was reported to be able to suppress the cell viability and colony formation ability in various cancer cells $(16,40,41)$. In the present study, we hypothesized that miR-101 inhibits cell growth and proliferation. As expected, ectopic expression of miR-101 suppressed the cell viability and cell proliferation of RB cell lines Y79 and WERI-RB1. Then we explored the potential mechanism attributed to the proliferation inhibitory role of miR-101 in RB cells. We found that ectopic expression of miR-101 resulted in cell cycle arrest in the G1 phase and promoted the cell apoptosis of RB cells. Our study confirmed the tumor-suppressive role of miR-101 in RB for the first time, and provides evidence for the potential usefulness of miR-101 in miRNA-based cancer therapy.

EZH2 is broadly overexpressed in aggressive solid tumors and displays the properties of an oncogene, as overexpression of EZH2 promotes cell proliferation, colony formation, migration and invasion in vivo and in vitro. $\mathrm{EZH} 2$ has been identified as a target of miR-101 by luciferase reporter assay in many types of cancer, but not in RB. In the present study, we examined the relationship of EZH2 and miR-101 in RB tissues and investigated whether miR-101 directly targets EZH2 in RB cells. We found that EZH2 was inversely associated with miR-101 expression in RB tissues. The mRNA level of EZH2 was also reduced in RB cells transfected with miR-101 mimics. Using luciferase assay, we found that miR-101 directly targeted EZH2 in RB cells. These result provide insight into the regulatory mechanism of miR-101 in RB. Downregulation of miR-101 in RB cells resulted in enhanced expression of EZH2, which consequently favored tumor progression.

In summary, we showed that miR-101 was downregulated and its target EZH2 was upregulated in RB tissues. Moreover, downregulated miR-101 and upregulated EZH2 were significantly associated with adverse clinicopathological and histopathological features of RB. Finally, we found that ectopic expression of miR-101 significantly reduced cell growth and proliferation in RB cells through directly targeting EZH2, which was associated with increased G1 phase arrest and cell apoptosis. These results suggest that miR-101 plays a role in inhibiting the development and progression of RB by targeting EZH2 and may potentially lead to a novel strategy of therapy for RB. 


\section{Acknowledgements}

The authors thank the local doctors and the patients who participated in our study.

\section{References}

1. Broaddus E, Topham A and Singh AD: Incidence of retinoblastoma in the USA: 1975-2004. Br J Ophthalmol 93: 21-23, 2009.

2. Siegel R, Naishadham D and Jemal A: Cancer statistics, 2013 CA Cancer J Clin 63: 11-30, 2013

3. Giacinti $\mathrm{C}$ and Giordano A: RB and cell cycle progression. Oncogene 25: 5220-5227, 2006.

4. Lillington DM, Kingston JE, Coen PG, et al: Comparative genomic hybridization of 49 primary retinoblastoma tumors identifies chromosomal regions associated with histopathology, progression, and patient outcome. Genes Chromosomes Cancer 36: 121-128, 2003.

5. Reis AH, Vargas FR and Lemos B: More epigenetic hits than meets the eye: microRNAs and genes associated with the tumorigenesis of retinoblastoma. Front Genet 3: 284, 2012.

6. Calin GA and Croce CM: MicroRNA signatures in human cancers. Nat Rev Cancer 6: 857-866, 2006.

7. Kumar MS, Lu J, Mercer KL, Golub TR and Jacks T: Impaired microRNA processing enhances cellular transformation and tumorigenesis. Nat Genet 39: 673-677, 2007.

8. Farazi TA, Hoell JI, Morozov P and Tuschl T: MicroRNAs in human cancer. Adv Exp Med Biol 774: 1-20, 2013.

9. Jansson MD and Lund AH: MicroRNA and cancer. Mol Oncol 6: 590-610, 2012

10. Ambros V: The functions of animal microRNAs. Nature 431: 350-355, 2004

11. Bueno MJ and Malumbres M: MicroRNAs and the cell cycle. Biochim Biophys Acta 1812: 592-601, 2011.

12. Kandalam MM, Beta M, Maheswari UK, Swaminathan S and Krishnakumar S: Oncogenic microRNA 17-92 cluster is regulated by epithelial cell adhesion molecule and could be a potential therapeutic target in retinoblastoma. Mol Vis 18 2279-2287, 2012

13. Dalgard CL, Gonzalez M, deNiro JE and O'Brien JM: Differential microRNA-34a expression and tumor suppressor function in retinoblastoma cells. Invest Ophthalmol Vis Sci 50: 4542-4551, 2009.

14. Wang J, Wang X, Wu G, Hou D and Hu Q: MiR-365b-3p, downregulated in retinoblastoma, regulates cell cycle progression and apoptosis of human retinoblastoma cells by targeting PAX6. FEBS Lett 587: 1779-1786, 2013.

15. Lee YS and Dutta A: The tumor suppressor microRNA let-7 represses the HMGA2 oncogene. Genes Dev 21: 1025-1030, 2007.

16. Varambally S, Cao Q, Mani RS, et al: Genomic loss of microRNA-101 leads to overexpression of histone methyltransferase EZH2 in cancer. Science 322: 1695-1699, 2008.

17. Chase A and Cross NC: Aberrations of EZH2 in cancer. Clin Cancer Res 17: 2613-2618, 2011.

18. Varambally S, Dhanasekaran SM, Zhou M, et al: The polycomb group protein $\mathrm{EZH} 2$ is involved in progression of prostate cancer. Nature 419: 624-629, 2002

19. Bracken AP, Pasini D, Capra M, Prosperini E, Colli E and Helin K: EZH2 is downstream of the pRB-E2F pathway, essentia for proliferation and amplified in cancer. EMBO J 22: 5323-5335, 2003.

20. Friedman JM, Liang G, Liu CC, et al: The putative tumor suppressor microRNA-101 modulates the cancer epigenome by repressing the polycomb group protein EZH2. Cancer Res 69: 2623-2629, 2009.

21. Wang HJ, Ruan HJ, He XJ, et al: MicroRNA-101 is downregulated in gastric cancer and involved in cell migration and invasion. Eur J Cancer 46: 2295-2303, 2010.
22. Smits M, Nilsson J, Mir SE, et al: miR-101 is down-regulated in glioblastoma resulting in EZH2-induced proliferation, migration, and angiogenesis. Oncotarget 1: 710-720, 2010.

23. Zhang JG, Guo JF, Liu DL, Liu Q and Wang JJ: MicroRNA-101 exerts tumor-suppressive functions in non-small cell lung cancer through directly targeting enhancer of zeste homolog 2 . J Thorac Oncol 6: 671-678, 2011.

24. Cao P, Deng Z, Wan M, et al: MicroRNA-101 negatively regulates Ezh2 and its expression is modulated by androgen receptor and HIF-1alpha/HIF-1beta. Mol Cancer 9: 108, 2010.

25. Wang L, Zhang X, Jia LT, et al: c-Myc-mediated epigenetic silencing of microRNA-101 contributes to dysregulation of multiple pathways in hepatocellular carcinoma. Hepatology 59: $1850-1863,2014$

26. Xu L, Beckebaum S, Iacob S, et al: MicroRNA-101 inhibits human hepatocellular carcinoma progression through EZH2 downregulation and increased cytostatic drug sensitivity. J Hepatol 60: 590-598, 2014.

27. Luo C, Merz PR, Chen Y, et al: MiR-101 inhibits melanoma cell invasion and proliferation by targeting MITF and EZH2. Cancer Lett 341: 240-247, 2013.

28. Nakagawa S, Sakamoto Y, Okabe H, et al: Epigenetic therapy with the histone methyltransferase EZH2 inhibitor 3-deazaneplanocin A inhibits the growth of cholangiocarcinoma cells. Oncol Rep 31: 983-988, 2014.

29. Yang YA and $\mathrm{Yu}$ J: EZH2, an epigenetic driver of prostate cancer. Protein Cell 4: 331-341, 2013.

30. Pushpavalli S, Ramaiah MJ, Srinivas $\mathrm{CH}$, et al: Effect of benzothiazole based conjugates in causing apoptosis by regulating p53, PTEN and MAP kinase proteins affecting miR-195a and miR-101-1. Cancer Cell Int 11: 36, 2011.

31. Ng SB, Yan J, Huang G, et al: Dysregulated microRNAs affect pathways and targets of biologic relevance in nasal-type natural killer/T-cell lymphoma. Blood 118: 4919-4929, 2011.

32. Hiroki E, Akahira J, Suzuki F, et al: Changes in microRNA expression levels correlate with clinicopathological features and prognoses in endometrial serous adenocarcinomas. Cancer Sci 101: 241-249, 2010.

33. Zhang Y, Guo X, Xiong L, et al: MicroRNA-101 suppresses SOX9-dependent tumorigenicity and promotes favorable prognosis of human hepatocellular carcinoma. FEBS Lett 586: 4362-4370, 2012

34. Yin J, Wang M, Jin C and Qi Q: miR-101 sensitizes A549 NSCLC cell line to CDDP by activating caspase 3 -dependent apoptosis. Oncol Lett 7: 461-465, 2014.

35. Xu Y, An Y, Wang Y, et al: miR-101 inhibits autophagy and enhances cisplatin-induced apoptosis in hepatocellular carcinoma cells. Oncol Rep 29: 2019-2024, 2013.

36. Lee KH, Chen YL, Yeh SD, et al: MicroRNA-330 acts as tumor suppressor and induces apoptosis of prostate cancer cells through E2F1-mediated suppression of Akt phosphorylation. Oncogene 28: 3360-3370, 2009.

37. Conkrite K, Sundby M, Mukai S, et al: miR-17 92 cooperates with RB pathway mutations to promote retinoblastoma. Genes Dev 25: 1734-1745, 2011.

38. Kong W, Yang H, He L, et al: MicroRNA-155 is regulated by the transforming growth factor beta/Smad pathway and contributes to epithelial cell plasticity by targeting RhoA. Mol Cell Biol 28: 6773-6784, 2008

39. Volinia S, Calin GA, Liu CG, et al: A microRNA expression signature of human solid tumors defines cancer gene targets. Proc Natl Acad Sci USA 103: 2257-2261, 2006.

40. Su H, Yang JR, Xu T, et al: MicroRNA-101, down-regulated in hepatocellular carcinoma, promotes apoptosis and suppresses tumorigenicity. Cancer Res 69: 1135-1142, 2009.

41. Li S, Fu H, Wang Y, et al: MicroRNA-101 regulates expression of the $v$-fos FBJ murine osteosarcoma viral oncogene homolog (FOS) oncogene in human hepatocellular carcinoma. Hepatology 49: 1194-1202, 2009. 